\title{
D-04
}

\section{EL DRENAJE FRANCÉS PREFABRICADO, ITINERARIO PARA LA OBTENCIÓN DEL MARCADO CE}

\author{
Madurell Fernández, Josep. (1) (P), Navarro Granados, Jordi (2) (P)
}

${ }^{1}$ Ingeniero Técnico de Obras Públicas. Especialidad Construcciones Civiles col. nº 9636 Responsable Técnico y Comercial del drenotube ${ }^{\circledR}$ en FUMOSO INDUSTRIAL S.A., areatecnica@drenotube.com.

${ }^{2}$ Ingeniero Escuela Superior de Ingeniería Industrial y Aeronáutica de Terrassa ETSEIAT. Jefe de proyecto, Departamento de Apoyo a la Innovación. ITeC-Instituto de Tecnología de la Construcción de Cataluña, inavarro@itec.cat.

\section{Resumen}

El drenaje tipo francés o también llamado subsuperficial contribuye en numerosos aspectos en la mejora de las condiciones para la producción agrícola. El más común de sus usos es captar y conducir el exceso de agua del terreno. A este uso van asociadas mas aportaciones como acortar tiempos tanto de siembra como de recolección, mejorar la aireación en la zona radicular contribuyendo a minimizar enfermedades y también para remediación de suelos con exceso de agroquímicos o sales naturales. En este trabajo se describe el proceso seguido para dar el respaldo técnico al nuevo sistema de drenaje francés prefabricado (DFP en adelante) que en vez de grava de cantera utiliza un granulado de EPS. Demostrando su idoneidad en el ámbito de la Unión Europea con la certificación CE según ETA 15/0201. El DFP tiene aplicación en drenaje agrícola y también en obras lineales como ferrocarril, carreteras, autovías y autopistas, obras de urbanización, edificación residencial e industrial, paisajismo, instalaciones deportivas de césped natural, artificial etc. (con ejemplos en todos estos casos). Los DFP con árido de EPS se inventaron en USA y tienen más de 25 años de experiencia, también se utiliza en Canadá por proximidad al mercado americano, en Japón como drenaje agrícola y más recientemente en Turquía, Rusia e Italia para construcción. En España la instalación agrícola más antigua tiene 6 años.

\section{1) Introducción}

El sector del drenaje a día de hoy no dispone de un marco normativo homogéneo en la UE; cada territorio tiene su forma de hacer y en cierta medida protege dicha tradición.

Existe la posibilidad de realizar de forma voluntaria, según se establece en el Reglamento de Productos de la Construcción (RPC 305/2011), el ETA (European Technical Assessment), que es la vía para conseguir el marcado CE para aquellos productos innovadores que carecen de norma armonizada o se desvían de una norma armonizada. El ETA recoge las prestaciones del producto en relación a los requisitos que el RPC 305/2011 considera para las obras de construcción. La conformidad del producto con estas prestaciones permite la obtención del marcado CE.

Así se realizó el ETA 15/0201 y el marcado CE de este DFP en el que se basa este trabajo. 
El DFP se presenta habitualmente en unidades de 3 o 6 metros en formato barra, que tiene un tubo de polietileno corrugado y ranurado en el centro y una capa de granulado de EPS rodeando al tubo. Está protegido por un geotextil filtrante y se cierra el conjunto con una malla de polietileno. Las barras se conectan mediante acopladores de plástico o manguitos.

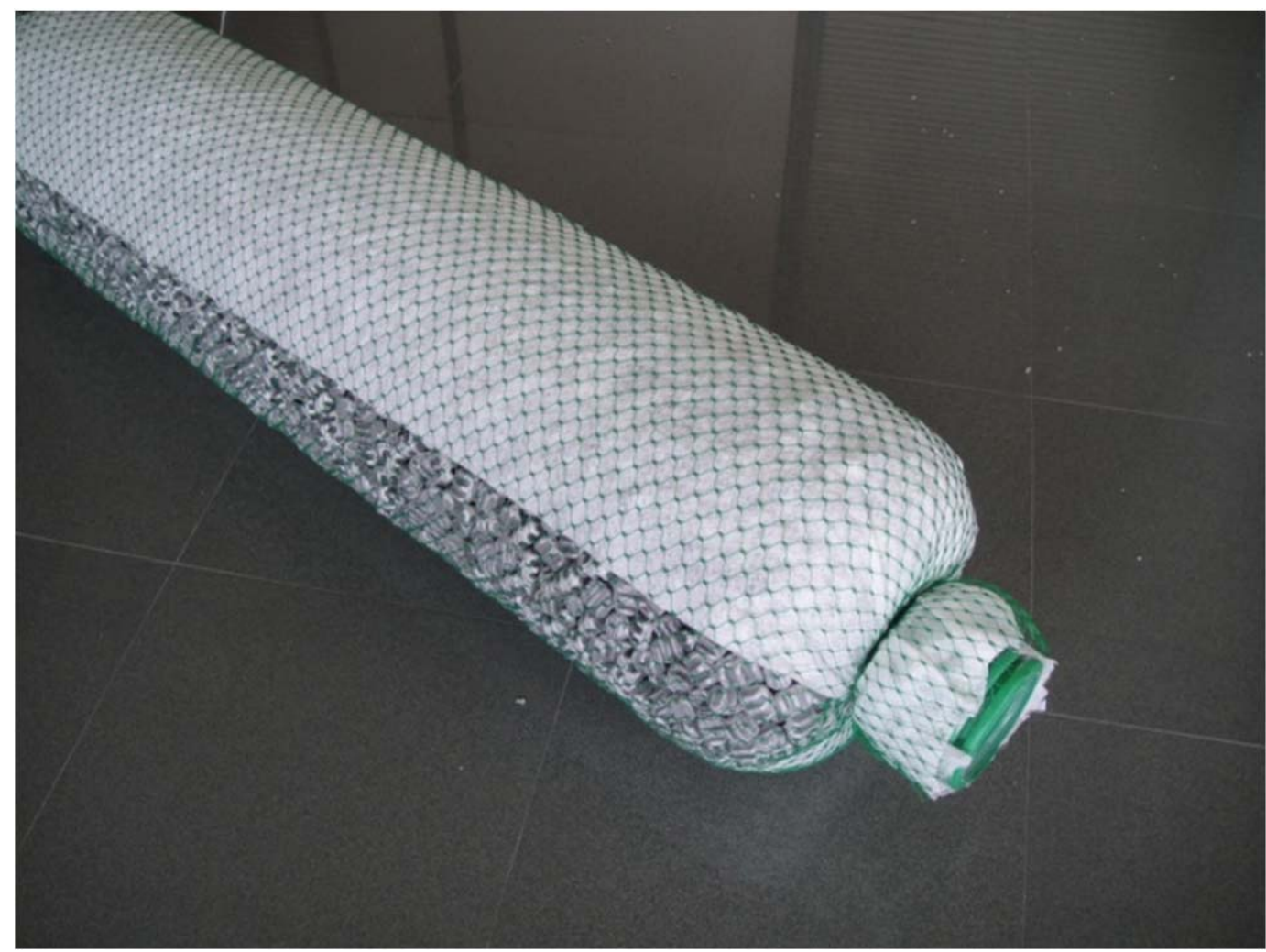

Imagen 1. Ejemplo de DFP

Las principales innovaciones del DFP, respecto a un drenaje tradicional son:

\section{En cuanto al dispositivo}

-geometría del dispositivo optimizada para mejorar el comportamiento hidráulico.

-el granulado de EPS de geometría específica que sustituye a la grava del drenaje francés convencional aporta:

-ligereza para fabricar un DFP para ser movido manualmente ( $>100$ veces más ligero que la grava), sin comprometer su componente resistente necesario para un amplio rango de usos para los que se ha ensayado ampliamente su comportamiento bajo presión.

- forma de granulado es más eficiente que la grava se estima en 30\%, por su forma y también porque el índice de huecos es del 50\%, superando ampliamente a cualquier material granular empleado en drenaje.

En las imágenes a continuación se detalla la forma más efectiva y principalmente utilizada, es el estándar americano y la que se ha certificado en Europa. Además se incluye el cuadro resumen con los resultados del estudio que aporta el fabricante americano en su documentación técnica, firmado por Mr. Donald Oderkirk, P.Eng, of BPI,pllc MEMPHIS, TENNESSEE de fecha 17/7/2001, en que analiza la capacidad drenante de varios materiales empleados en drenajes, de forma aislada y en conjunto. Tanto el planteamiento como los resultados son valorados por su facilidad de comprensión y el haber podido contrastar esos resultados con los ensayos realizados para este caso en la U.E. 


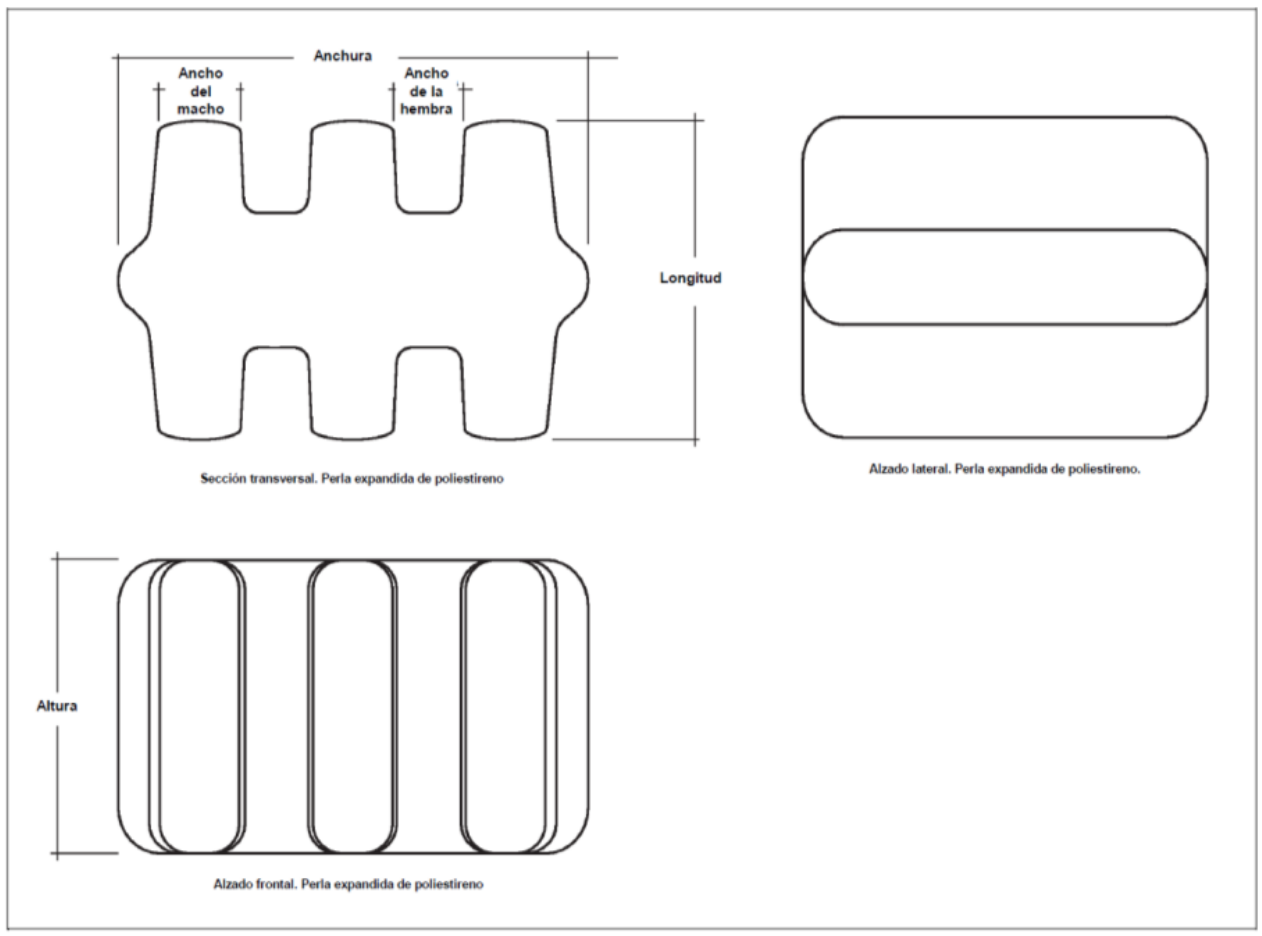

Imagen 2. Granulado de EPS

GRANULADO DE EPS
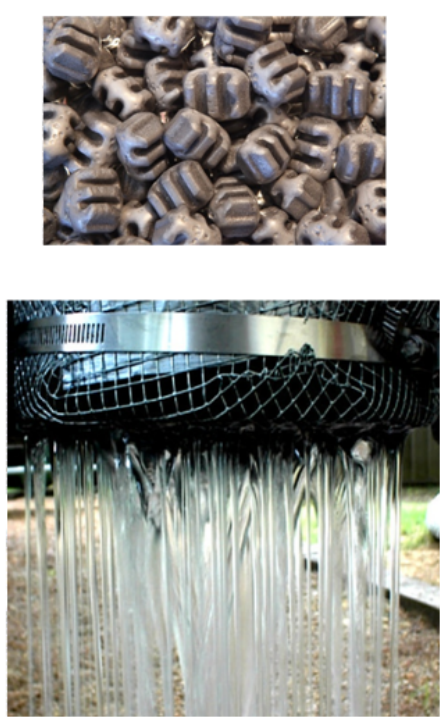

Caudal: 0,90 litros/segundo 30 veces más drenante que la gravilla 50 veces más drenante que la arena lavada
GRAVILLA
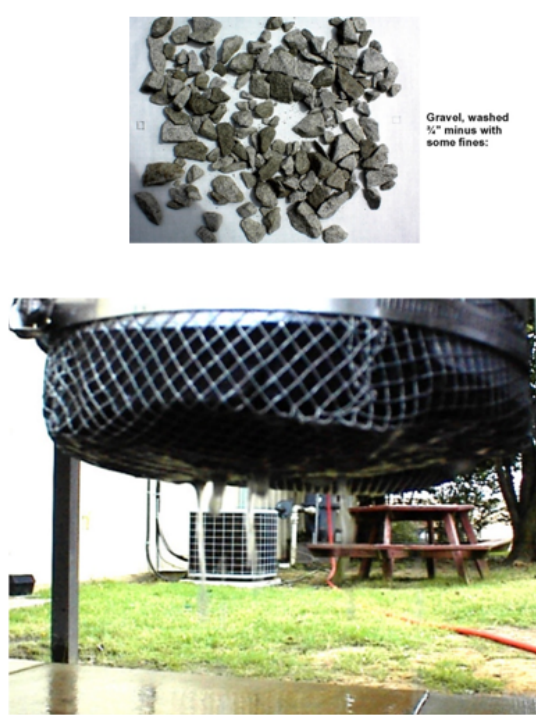

Caudal: 0,03 litros/segundo
ARENA LAVADA
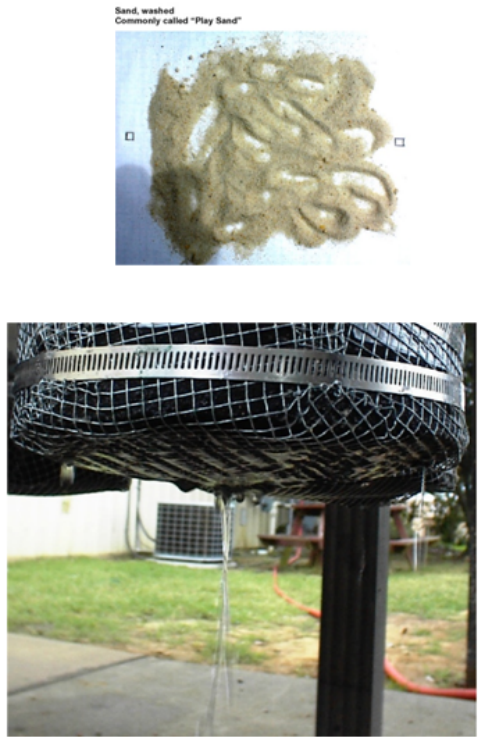

Caudal: 0,018 litros/segundo

Imagen 3. Comparativo de la conductividad hidráulica de materiales porosos para drenajes

\section{En cuanto a su instalación}

-Sistema modular para instalación manual con rendimiento de instalación 50\% mejor.

- Sistema que proporciona máxima calidad y durabilidad, con el único compromiso de ejecutar la excavación según proyecto. Muy adecuado para agricultura pues tanto le vale a un particular como a un instalador. 


\section{2) Itinerario para alcanzar el CE de los productos innovadores}

Como producto innovador sin ningún precedente normativo armonizado a nivel europeo, la consecución del marcado CE para el drenaje francés prefabricado (DFP) implicó, según lo estipulado en el Artículo 21.1 c) del Reglamento 305/2011:

- La elaboración y aprobación europea de un DEE-Documento de Evaluación Europeo: este documento constituye la guía de evaluación europea de referencia para esta tipología de productos, que fue finalmente publicada en el DOUE (10/07/15). Este DEE 280001-00-0704 Preassembled line unit used for drainage or infiltration se encuentra a disposición de otros fabricantes de estos productos que deseen obtener el marcado CE.

- La elaboración del ETA 15/0201 para el drenaje certificado.

Esto permitió al fabricante realizar el marcado CE y Declaración de Prestaciones del producto. El marcado CE facilita el acceso del producto a los mercados europeos y da confianza técnica a los usuarios acerca de sus prestaciones.

A continuación se desarrollan con un poco más de detalle las bases y criterios conceptuales del Reglamento 305/2011 y se describe la metodología para alcanzar el marcado CE de un producto innovador.

\section{$\underline{\text { Introducción }}$}

Los productos de construcción se rigen por lo estipulado por el Reglamento de Productos de la Construcción (UE) 305/2011, que fija las condiciones para la comercialización de los productos de construcción y para la determinación según reglas armonizadas de las prestaciones de los productos y su posterior declaración.

Tiene por lo tanto la finalidad última de hacer posible un mercado único de productos de construcción en la Unión Europea, que comparta un lenguaje técnico único armonizado en cuanto a las prestaciones de los productos.

Se entiende como producto de construcción aquel que vaya a formar parte de las obras de construcción (lo cual incluye las obras de edificación y la ingeniería civil) de forma permanente y cuyas prestaciones influyan en las prestaciones de la obras relacionadas con los requisitos básicos de las mismas.

Los requisitos básicos de las obras contemplados por el Reglamento son:

(1) Resistencia mecánica y estabilidad

(2) Seguridad en caso de incendio

(3) Higiene, salud y medio ambiente

(4) Seguridad y accesibilidad de utilización

(5) Protección frente al ruido

(6) Ahorro de energía y aislamiento térmico

(7) Utilización sostenible de recursos naturales

Asimismo el Reglamento establece cuáles son los agentes económicos del proceso de comercialización (fabricantes, representantes autorizados, importadores, distribuidores) y las obligaciones de cada uno de ellos.

Todo ello se traduce en que los fabricantes deben proceder, siguiendo las metodologías armonizadas, al Marcado CE de sus productos y a la emisión de la Declaración de prestaciones (DoP) para la puesta en el mercado de sus productos.

Cabe no obstante diferenciar los dos casos siguientes: 
- Productos de construcción totalmente cubiertos por una norma armonizada existente: el fabricante está obligado a proceder al Marcado CE con arreglo a lo estipulado en la norma armonizada en cuestión.

- Productos de construcción innovadores, que carecen de norma armonizada o que se desvían de una norma armonizada existente: el fabricante no está obligado a proceder al Marcado CE. No obstante, si desea acceder al Marcado CE, esto es siempre posible por una de las vías que establece el artículo 21.1 cláusulas (b) y (c), es decir, por la vía de la elaboración de un documento ETE-Evaluación Técnica Europea. El ETE podría definirse, en términos informales, como una "norma hecha a medida de los requisitos aplicables al producto y usos considerados", si bien, y a diferencia de una norma, el ETE es nominal (es para un producto comercial de un determinado fabricante).

La no obligatoriedad del marcado CE para estos productos innovadores y no normados no debe entenderse como que el fabricante de los mismos queda eximido de toda necesidad de demostrar las prestaciones de su producto. Por el contrario, debe demostrar frente al mercado y de forma fehaciente las prestaciones de su producto, máxime cuando éste puede ser un producto innovador que carece de precedentes técnicos y, por lo tanto, sus usuarios no disponen de referencias acerca de sus prestaciones y comportamiento técnico.

Para dar respuesta a esta necesidad de demostrar las prestaciones de su producto, el fabricante puede optar por un documento de evaluación de la idoneidad de ámbito nacional o puede optar por la certificación de ámbito europeo por la vía del Marcado CE basado en un ETE. De ahí que hablemos que el marcado CE vía ETE es voluntario para productos innovadores y no normados.

Naturalmente la certificación de ámbito europeo dará acceso a un mercado más amplio que una certificación de ámbito español, y sería la vía preferente en el caso que el fabricante se esté planteando comercializar el producto fuera de España.

\section{El marcado CE de productos innovadores y no normados por la vía del ETE}

Como ya se ha avanzado en la introducción, los productos huérfanos de norma europea armonizada pueden acceder al marcado CE por la vía de la elaboración de una Evaluación Técnica Europea-ETE, elaborada por parte de un Organismo de Evaluación Técnica-OET, oficialmente autorizado ${ }^{1}$.

Para la elaboración del ETE el fabricante se puede encontrar con los dos casos siguientes:

- El producto y usos objeto de la petición del ETE están cubiertos por una guía de evaluación europea ya existente, llamada DEE-Documento de Evaluación Europeo (caso b) del artículo 21.1 del Reglamento).

- El producto y usos objeto de la petición del ETE no están cubiertos por una guía de evaluación europea existente, por lo que es preciso desarrollar y consensuar a nivel europeo un DEE específico para la petición de ETE que formula el fabricante (caso c) del artículo 21.1 del Reglamento).

El primer caso implica la elaboración directa del ETE con arreglo al DEE existente, mientras que el segundo requiere de la elaboración previa de un DEE específico, hecho lo cual se aborda el ETE. Naturalmente el segundo caso implica un plazo de ejecución superior.

EI DEE lo desarrolla el OET que recibe la petición de ETE del fabricante, siendo este desarrollo llevado a cabo de forma colegiada con el resto de OETs miembros de la EOTA ${ }^{2}$ y

\footnotetext{
${ }^{1}$ El ITeC es un organismo autorizado desde el año 1997 para la emisión del ETE en todas las áreas de producto del Reglamento de Productos 305/2011.

${ }^{2}$ EOTA: European Organisation for Technical Assessment.
} 
según las directrices de la Comisión Europea, de acuerdo con la metodología del Anexo II del Reglamento.

En este DEE se tienen en cuenta los requisitos establecidos en las reglamentaciones nacionales de construcción aplicables al producto y uso en cuestión de los países europeos en los que el fabricante desea comercializar el producto en cuestión, así como los métodos armonizados para la verificación de dichos requisitos. Estos métodos pueden ser ensayos de laboratorio, verificaciones por cálculo o adopción de valores tabulados, en todos los casos tratándose de métodos que se han adoptado a nivel europeo como metodologías armonizadas.

EI DEE es un documento de carácter confidencial durante su elaboración y de carácter público una vez se aprueba el mismo, momento en el cual el DEE se publica en el DOUE (Diario Oficial de la Unión Europea) y pasa a estar disponible para otros fabricantes de productos incluidos en el alcance del DEE que deseen conseguir el marcado CE de sus productos.

\section{Las tareas de Verificación de la Constancia de Prestaciones}

Independientemente de si el producto dispone de norma armonizada o ha requerido de la elaboración de un ETE específico para él por carecer de norma armonizada de referencia, la última etapa para la consecución del marcado CE implica llevar a cabo las tareas de Verificación de la Constancia de Prestaciones (VCP).

En función del sistema de VCP que haya dictado la Comisión Europea para el producto (lo cual queda detallado en la norma o ETE de referencia), el grado de intervención de un organismo notificado será mayor o menor. Como regla general, en productos relacionados con el cumplimiento de requisitos de seguridad, el sistema VCP tiende a ser el más exigente (sistema $101+$ ), mientras que en el lado opuesto, para productos no relacionados 0 débilmente relacionados con el cumplimiento de requisitos de seguridad el sistema VCP tiende a ser el menos exigente (sistema 4).

Bajo cualquiera de los sistemas (desde el 1+ al 4) el fabricante debe implementar un sistema de control de producción en fábrica que asegure la constancia de las prestaciones del producto que el fabricante declarará frente al mercado.

La intervención de organismos notificados en tareas de certificación se limita a los sistemas $(1,1+$ y 2$)$, mientras que bajo el sistema 3 interviene un laboratorio notificado. Bajo el sistema 4 el fabricante puede llevar a cabo los ensayos por sus propios medios sin recurrir a un organismo notificado, siempre que estos medios respondan a los requisitos de la especificación armonizada de referencia.

Hechas estas tareas de VCP según el sistema que aplique en cada caso, el fabricante podrá:

- Marcar su producto CE.

- Emitir la Declaración de Prestaciones de su producto (DoP).

Esta DoP constituye el documento con carácter legal con el que el fabricante declara formalmente las prestaciones de su producto frente al mercado.

El marcado CE de los Drenajes franceses prefabricados (DFP) según el DEE 280001-000704 Preassembled line unit used for drainage or infiltration

EI DEE de referencia para el marcado CE de los DFP contempla la evaluación de los siguientes aspectos principales del comportamiento del producto drenante: 
- Deformación del producto frente a las cargas de uso (cargas permanentes del terreno y sobrecargas de uso debidas al tránsito de vehículos), así como deformación a largo plazo frente a las cargas permanentes (fluencia).

- Capacidad drenante del producto, tanto en situación de carga como en condiciones de ausencia de carga mecánica sobre el drenaje.

- Prestaciones del geotextil (resistencia al punzonamiento estático y dinámico).

- Durabilidad del drenaje.

En consecuencia, el marcado CE de un DFP ofrece al técnico usuario del producto la información técnica necesaria para poder valorar la adecuación del mismo a las condiciones de uso previstas en el proyecto, con arreglo a los requisitos reglamentarios que sean de aplicación al proyecto en cuestión.

\section{3) Conclusiones}

Un buen drenaje es tan importante o más que un buen riego, pero sigue siendo la asignatura pendiente en muchos lugares. Con el DFP el sector agrícola tiene la herramienta para mejorar las condiciones de la producción agrícola, desde poner a producir zonas no útiles por exceso de agua del terreno que provoca asfixia radicular a usos asociados a productividad como acortar tiempos tanto de siembra como de recolección, mejorar la aireación de la zona radicular contribuyendo a minimizar enfermedades y también para la remediación de suelos con exceso de agroquímicos o sales naturales.

La garantía de un buen drenaje no debe ser inferior a 25 años, el ETA 15/0201 asegura el cumplimiento de esta premisa.

El marcado CE, que es obligatorio para los productos que disponen de norma europea armonizada, es de carácter voluntario para los productos que carecen de norma. Para éstos, el marcado CE es siempre posible, y ofrece a los fabricantes una respuesta a los requisitos reglamentarios con los que se encuentran en Europa. Por ello el marcado CE facilita la entrada del producto innovador en estos mercados europeos, evitando llevar a cabo otras certificaciones nacionales país a país, necesarias en caso de carecer del marcado CE.

La inversión para alcanzar el marcado CE implica un notable esfuerzo financiero y técnico para la empresa; es muy importante anticiparse tanto como sea posible al requisito que se va a encontrar el producto en el mercado, para iniciar el procedimiento de certificación lo antes posible.

\section{4) Agradecimientos}

- La ASOCIACION ESPAÑOla DE RIEgos Y DRENAJES (AERYD), por su amabilidad y gran colaboración, permitiendo la presentación de este trabajo.

- Laboratorio AITEX (Asociación de Investigación de la Industria Textil), especialmente a los interlocutores durante las 2 fases de ensayos realizados Dña. Begoña Francés y D. Axel Ferrando.

- Laboratorio CECAM (centro de estudios de la construcción y análisis de materiales), por el trabajo de D. Lluís Rodríguez y la estrecha colaboración en desarrollar ensayos nuevos y la mejora continua obtenida.

- ITeC-Instituto de Tecnología de la Construcción de Cataluña, agente EOTA por su profesionalidad y enorme paciencia. D. Jordi Roher y D. Jordi Navarro. 


\section{5) Bibliografía}

- EOTA-European Organization for Technical Assessment (2014), Preassembled line unit used for drainage or infiltration, Official Journal of the European Union, Brussels.

- European Commission (2011), Regulation (EU) No 305/2011 of the European Parliament and the Council of 9 March 2011 laying down harmonised conditions for the marketing of construction products, Official Journal of the European Union 4.4.2011 L 88/5, Brussels.

- Flórez, P. (2015) Revista Aimcra no 121, 52-54

- Oderkirk, D. (2001). HYDRAULIC CONDUCTIVITY. An Evaluation of Drainage Media in Controlled Conditions, of BPI,pllc MEMPHIS, TENNESSEE 